\title{
SLR: Path-Sensitive Analysis through Infeasible-Path Detection and Syntactic Language Refinement.
}

\author{
Gogul Balakrishnan ${ }^{1}$, Sriram Sankaranarayanan ${ }^{1}$, Franjo Ivančić $^{1}, \mathrm{Ou} \mathrm{Wei}^{2}$, \\ and Aarti Gupta ${ }^{1}$ \\ 1 NEC Laboratories America, Princeton, NJ, USA. \\ 2 University of Toronto \\ \{bgogul, srirams, ivancic, agupta\}@nec-labs.com, owei@cs.toronto.edu
}

\begin{abstract}
We present a technique for detecting semantically infeasible paths in programs using abstract interpretation. Our technique uses a sequence of path-insensitive forward and backward runs of an abstract interpreter to infer paths in the control flow graph that cannot be exercised in concrete executions of the program.

We then present a syntactic language refinement (SLR) technique that automatically excludes semantically infeasible paths from a program during static analysis. SLR allows us to iteratively prove more properties. Specifically, our technique simulates the effect of a path-sensitive analysis by performing syntactic language refinement over an underlying pathinsensitive static analyzer. Finally, we present experimental results to quantify the impact of our technique on an abstract interpreter for $\mathrm{C}$ programs.
\end{abstract}

\section{Introduction}

Static analysis techniques compute over-approximations of the reachable states for a given program. The theory of abstract interpretation is used to compute such an over-approximation as a fixpoint in a suitably chosen abstract domain [5, 6 . The degree of approximation as well as the scalability can be traded-off through a judicious choice of an abstract domain. However, the presence of approximations can cause the analysis to report false positives. Such false positives can be avoided, in part, using techniques such as path-sensitive analysis, disjunctive completion, and various forms of refinements $[1,7,8,11,13,17,22]$.

In practice, many syntactic paths in the control-flow graph (CFG) representation of the program are semantically infeasible, i.e, they may not be traversed by any execution of the program. Reasoning about the infeasibility of such paths is a key factor in performing accurate static analyses for checking properties such as correct API usage, absence of null-pointer dereferences and uninitialized use of variables, memory leaks, and so on. Our previous experience with building path-sensitive abstract interpreters [22] also indicates that the benefit of added path sensitivity to static analysis seems to lie mostly in the identification and elimination of semantically infeasible paths. 


$$
\begin{aligned}
& 0: \text { if }(\mathrm{x}>0) \\
& 1: \quad \mathrm{f}:=1 ; \\
& 2: \text { else } \\
& \text { 3: } \quad \mathrm{f}:=0 \\
& \text { 4: } \mathrm{y}:=\mathrm{x} ; \\
& \text { 5: if }(\mathrm{f}>0) \\
& 6: \quad \operatorname{ASSERT}(\mathrm{y}>=0)
\end{aligned}
$$

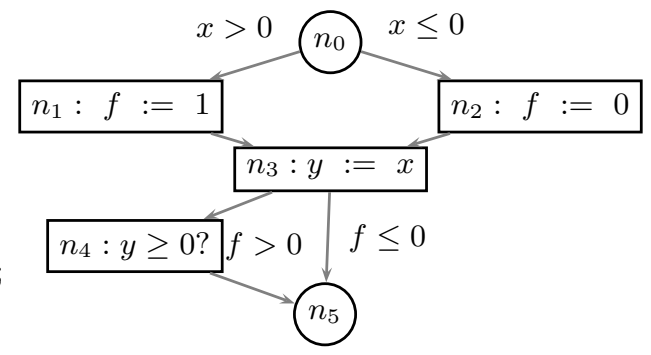

Fig. 1. An example program (left) along with its CFG representation (right). (Node numbers do not correspond to line numbers.)

This paper presents two main contributions. We present a technique based on path-insensitive abstract interpretation to infer semantically infeasible paths in the CFG. Secondly, we use a syntactic language refinement scheme for pathsensitive analysis by iteratively removing infeasible paths from the CFG.

Our technique for inferring semantically infeasible paths performs a sequence of many forward and backward runs using a path-insensitive abstract interpreter. We first present infeasibility theorems that use the results of forward/backward fixpoints obtained starting from different initial conditions to characterize paths in the CFG that are semantically infeasible. We then present techniques that enumerate infeasible paths using propositional SAT solvers without repeating previously enumerated paths.

The infeasible paths detected by our technique are excluded from the CFG using syntactic language refinement. Starting with the syntactic language defined by the set of all syntactically valid CFG paths, we remove semantically infeasible paths from the syntactic language to obtain refinement of the original language. Using a path-insensitive analysis over the refined syntactic language effectively incorporates partial path-sensitivity into the analysis, enabling us to prove properties that are beyond the reach of path-insensitive analyses.

Example 1. The program in Fig. 1 depicts a commonly occurring situation in static analysis. Abstract interpretation using the polyhedral abstract domain is unable to prove the property since it loses the correlation between $f$ and $x$ by computing a join at node $n_{3}$. On the other hand, our techniques allow us to prove using path-insensitive analysis that any semantically feasible path from node $n_{0}$ to node $n_{4}$ cannot pass through node $n_{2}$. Syntactic language refinement removes this path from the CFG, and performs a new path-insensitive analysis on the remaining paths in the CFG. Such an analysis maintains the correlation between $x$ and $f$ at node $n_{3}$ and successfully proves the property.

The F-SofT tool checks C programs for invalid pointer accesses, buffer overflows, memory leaks, incorrect usage of APIs, and arbitrary safety properties specified by a user [14]. We use the techniques described in the paper to improve the path-insensitive analysis used inside the F-SoFT tool to obtain the effects 
of path-sensitive analysis. The resulting analyzer proves more properties with a reasonable resource overhead.

Related Work. Path-sensitive analyses help minimize the impact of the join operation at the merge points in the program. A completely path-sensitive analysis is forbiddingly expensive. Many heuristic schemes achieve partial path-sensitive solutions that selectively join or separate the contributions due to different paths using logical disjunctions [8-10,13,17,22]. While path-sensitive analysis techniques modify the analysis algorithm, it is possible to achieve path-sensitivity by modifying the abstract domain using powerset extensions $[1,16]$. Finally, refinement-based techniques can modify the analysis algorithm or the domain itself on demand, based on the failure to prove a property. Gulavani and Rajamani iteratively refine the analysis algorithm by modifying analysis parameters such as widening delays and using weakest preconditions inside a powerset domain [11]. Cousot, Ganty, and Raskin present a fixpoint-based refinement technique that refines an initial Moore-closed abstract domain by adding predicates based on preconditions computed in the concrete domain [7].

Our technique for detecting infeasible paths relies on repeated forward and backward fixpoints. Similar ideas on using path-insensitive analyses to reason about specific program paths have appeared in the context of abstract debugging and semantic slicing $[4,20]$, which help to interactively zero-in on bugs in code by generating invariants, intermediate assertions, and weakest preconditions.

The use of infeasible paths to refine data-flow analysis has been considered previously by Bodik et al. [3]. However, our approach is more general in many ways: (a) we use abstract interpretation in a systematic manner to handle loops, conditions, procedures, and so on without sacrificing soundness, (b) the underlying analysis used to detect infeasible paths in our approach is itself pathinsensitive, which makes it possible to apply our approach on a whole-program basis without requiring much overhead or depth cutoffs.

Ngo and Tan [19] use simple syntactic heuristics to detect infeasible paths in programs that are used in test generation. Surprisingly, their approach seems to detect many infeasible paths using relatively simplistic methods. Such lightweight approaches can also be used as a starting point for syntactic language refinement.

\section{Preliminaries}

Throughout this paper, we consider single-procedure (while) programs over integer variables. Our results also extend to programs with many procedures and complex datatypes. We use control-flow graphs (CFG) to represent programs. A CFG is a tuple $\left\langle N, E, V, \mu, n_{0}, \varphi_{0}\right\rangle$, where $N$ is a set of nodes, $E \subseteq N \times N$ is a set of edges, $n_{0} \in N$ is an initial location, $V$ is a set of integer-valued program variables, and $\varphi_{0}$ specifies an initial condition over $V$ that holds at the start of the execution. Each edge $e \in E$ is labeled by a condition or an update $\mu(e)$.

A state of the program is a map $s: V \rightarrow Z$, specifying the value of each variable. Let $\Sigma$ be the universe of all valuations to variables in $V$. A program is assumed to start from the initial location $n_{0}$ and a state $s \in \llbracket \varphi_{0} \rrbracket$. 
The semantics of an edge $e \in E$ is given by the (concrete) strongest postcondition post $(e, S)$ and the (concrete) weakest precondition (backward postcondition) pre $(e, S)$ for sets $S \subseteq \Sigma$. The operator post : $E \times 2^{\Sigma} \rightarrow 2^{\Sigma}$ yields the smallest set of states reachable upon executing an edge from a given set of states $S$, while the pre : $E \times 2^{\Sigma} \rightarrow 2^{\Sigma}$ yields the largest set $T$ such that $t \in T$ iff post $(e,\{t\}) \subseteq S$. The pre operator is also the post for the "reverse" semantics for the edge $e$.

A flow-sensitive concrete map $\eta: N \rightarrow 2^{\Sigma}$ associates a set of states with each node in the CFG. We denote $\eta_{1} \subseteq \eta_{2}$ iff $\forall n \in N, \eta_{1}(n) \subseteq \eta_{2}(n)$.

Forward Propagation. ${ }^{3}$ The forward-propagation operator $\mathfrak{F}$ takes a concrete map $\eta: N \rightarrow 2^{\Sigma}$ and returns a new concrete map $\eta^{\prime}: N \rightarrow 2^{\Sigma}$ such that

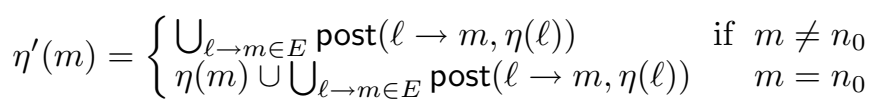

A map $\eta$ is inductive iff (a) $\eta\left(n_{0}\right) \supseteq \llbracket \varphi_{0} \rrbracket$ and (b) $\eta \supseteq \mathfrak{F}(\eta)$. In other words, if $\eta$ is inductive, it is also a post fixpoint of $\mathfrak{F}$. Since $\mathfrak{F}$ is a monotone operator, the least fixpoint always exists due to Tarski's theorem.

Given a CFG, a property consists of a pair $\langle n, \varphi\rangle$ where $n \in N$ is a node, and $\varphi$ is a first-order assertion representing a set of states. Associated with each property $\langle n, \varphi\rangle$, the CFG has a node $n_{E} \in N$ and an edge $\left(n \rightarrow n_{E}\right) \in E$ with condition $\varphi_{E}$, where $\varphi_{E}$ is $\neg \varphi$. The pair $\left\langle n_{E}, \varphi_{E}\right\rangle$ is referred to as the error configuration for property $\langle n, \varphi\rangle$. A property $\langle n, \varphi\rangle$ is verified if the error configuration $\left\langle n_{E}, \varphi_{E}\right\rangle$ is unreachable, i.e., if $\eta\left(n_{E}\right)=\emptyset$ for an inductive map $\eta$.

The least fixpoint of a monotone operator $\mathfrak{F}$ can be computed using Tarski iteration. Starting from the initial map $\eta^{0}$, such that $\eta^{0}\left(n_{0}\right)=\llbracket \varphi_{0} \rrbracket$ and $\eta^{0}(m)=\emptyset$ for all $m \neq n_{0}$, we iteratively compute $\eta^{i+1}=\mathfrak{F}\left(\eta^{i}\right)$ until a fixpoint is reached. Unfortunately, this process is computationally infeasible, especially if the program is infinite state.

To analyze programs tractably, abstract interpretation is used to compute post fixpoints efficiently. An abstract domain consists of a lattice $\langle L, \sqsubseteq, \sqcup, \sqcap\rangle$, along with the abstraction map $\alpha: 2^{\Sigma} \rightarrow L$ and the concretization map $\gamma$ : $L \rightarrow 2^{\Sigma}$. Each abstract object $a \in L$ is associated with a set of states $\gamma(a) \subseteq \Sigma$. The maps $\alpha$ and $\gamma$ together provide a Galois Connection between the concrete lattice $2^{\Sigma}$ and the abstract lattice $L$. The abstract counterparts for the union $(\cup)$ and intersection $(\cap)$ are the lattice join $(\sqcup)$ and lattice meet $(\sqcap)$ operators, respectively. Finally, the concrete post-conditions and concrete preconditions have the abstract counterparts post $_{L}$ and pre $_{L}$ in the abstract lattice $L$. A flowsensitive abstract map $\eta^{\sharp}: N \rightarrow L$ associates each node $n \in N$ to an abstract object $\eta^{\sharp}(n) \in L$. As before, $\eta_{1}^{\sharp} \sqsubseteq \eta_{2}^{\sharp}$ iff $\forall n \in N, \eta_{1}^{\sharp}(n) \sqsubseteq \eta_{2}^{\sharp}(n)$.

The forward propagation operator $\mathfrak{F}$ can be generalized to a monotone operator $\mathfrak{F}_{L}: \eta^{\sharp} \mapsto \eta^{\sharp}$ in the lattice $L$ such that:

$$
\eta^{\sharp}(m)= \begin{cases}\bigsqcup_{\ell \rightarrow m \in E} \operatorname{post}_{L}\left(\ell \rightarrow m, \eta^{\sharp}(\ell)\right) & \text { if } m \neq n_{0} \\ \eta^{\sharp}(m) \sqcup \bigsqcup_{\ell \rightarrow m \in E} \operatorname{post}_{L}\left(\ell \rightarrow m, \eta^{\sharp}(\ell)\right) & m=n_{0}\end{cases}
$$

\footnotetext{
${ }^{3}$ Our presentation assumes that the initial node $n_{0}$ may have predecessors.
} 
For a given program, abstract interpretation starts with the initial map $\eta_{0}^{\sharp}$, where $\eta_{0}^{\sharp}\left(n_{0}\right)=\alpha\left(\llbracket \varphi_{0} \rrbracket\right)$ and $\eta_{0}^{\sharp}(m)=\perp$ for all $m \neq n_{0}$. The process converges to a fixpoint $\eta_{F}^{\sharp}$ in $L$ if $\eta_{i+1}^{\sharp} \sqsubseteq \eta_{i}^{\sharp}$. Furthermore, its concretization $\gamma \circ \eta_{F}^{\sharp}$ is inductive (post fixpoint) on the concrete lattice. In practice, widening heuristics enforce convergence of the iteration in lattices that do not satisfy the ascending chain condition.

Backward Propagation. An alternative to verifying a property with error configuration $\left\langle n_{E}, \varphi_{E}\right\rangle$ is backward propagation using the backward propagation operator $\mathcal{B}$, which takes a map $\eta: N \rightarrow 2^{\Sigma}$ and returns a new map $\eta^{\prime}: N \rightarrow 2^{\Sigma}$ :

$$
\eta^{\prime}(\ell)= \begin{cases}\eta(\ell) \bigcup_{\ell \rightarrow m \in E} \operatorname{pre}(\ell \rightarrow m, \eta(m)) & \text { if } \ell=n_{E} \\ \bigcup_{\ell \rightarrow m \in E} \operatorname{pre}(\ell \rightarrow m, \eta(m)) & \text { otherwise }\end{cases}
$$

For an error configuration $\left\langle n_{E}, \varphi_{E}\right\rangle$, we compute the least fixpoint of $\mathcal{B}$ starting with the initial map $\eta^{0}$ such that $\eta^{0}\left(n_{E}\right)=\llbracket \varphi_{E} \rrbracket$ and $\eta^{0}(m)=\emptyset$ for all $m \neq n_{E}$. A map $\eta$ is a post fixpoint of the operator $\mathcal{B}$, if $\mathcal{B}(\eta) \subseteq \eta$. The property can be verified if $\eta\left(n_{0}\right) \cap \llbracket \varphi_{0} \rrbracket=\emptyset$, which establishes that it is not possible to reach an error state in $\llbracket \varphi_{E} \rrbracket$ at node $n_{E}$ from a state in $\llbracket \varphi_{0} \rrbracket$ at the initial node $n_{0}$.

Analogous to forward propagation, one may compute a backward (post) fixpoint map $\eta_{B}^{\sharp}$ in an abstract domain $L$ by extending the operator $\mathcal{B}$ to the lattice $L$ using the precondition map pre ${ }_{L}$ to yield $\mathcal{B}_{L}$. The fixpoint map $\eta_{B}^{\sharp}$ computed using $\mathcal{B}_{L}$ can be used to verify properties.

\section{Infeasible-Path Detection}

We now characterize infeasible paths in the program using abstract interpretation. Rather than focus on individual paths (of which there may be infinitely many), our results characterize sets of infeasible paths, succinctly. For the remainder of the section, we assume a given abstract domain $\langle L, \sqsubseteq, \sqcup, \sqcap\rangle$ (or even a combination of many abstract domains) that defines the forward operator $\mathfrak{F}_{L}$ and the backward operator $\mathcal{B}_{L}$. These operators transform initial maps $\eta_{F_{0}}^{\sharp}$ $\left(\eta_{B_{0}}^{\sharp}\right)$ into post fixpoints $\eta_{F}^{\sharp}\left(\eta_{B}^{\sharp}\right)$ using abstract forward (backward) propagation. The fixpoint maps $\eta_{F}^{\sharp}$ and $\eta_{B}^{\sharp}$ are concretized to yield maps $\eta_{F}=\gamma \circ \eta_{F}^{\sharp}$ and $\eta_{B}=\gamma \circ \eta_{B}^{\sharp}$, respectively. Therefore, we present our results in the concrete domain based on concretized fixpoint maps $\eta_{F}$ and $\eta_{B}$.

Consider a node $n \in N$ and a set $\llbracket \varphi \rrbracket$. We define a basic primitive called state-set projection that projects $\langle n, \varphi\rangle$ onto another node $m \in N$ in the CFG as follows: (a) We compute the forward fixpoint map $\eta_{F}$ and the backward fixpoint map $\eta_{B}$ starting from the following initial map:

$$
\eta_{0}^{\langle n, \varphi\rangle}(\ell)=\left\{\begin{array}{l}
\llbracket \varphi \rrbracket, \quad \ell=n \\
\perp, \quad \text { otherwise }
\end{array}\right.
$$

(b) The set $\eta_{F}(m)$ is a forward projection and $\eta_{B}(m)$ is a backward projection of $\langle n, \varphi\rangle$ onto $m$. 
Definition 1 (State-set Projection). A forward projection of the pair $\langle n, \varphi\rangle$ onto a node $m$, denoted $(\langle n, \varphi\rangle \stackrel{L}{\hookrightarrow} m)$ is the set $\eta_{F}(m)$, where $\eta_{F}$ is a forward (post) fixpoint map starting from the initial map $\eta_{0}^{\langle n, \varphi\rangle}$.

Similarly, a backward projection of the pair $\langle n, \varphi\rangle$ back onto $m$, denoted ( $m \stackrel{L}{\longleftarrow}\langle n, \varphi\rangle)$ is the set $\eta_{B}(m)$, where $\eta_{B}$ is the backward fixpoint starting from the initial map $\eta_{0}^{\langle n, \varphi\rangle}$.

Forward and backward state-set projections are not unique. They vary, depending on the specific abstract interpretation scheme used to compute them. The projection of a node $n$ onto itself yields the assertion true.

Lemma 1. Let $\varphi_{F}:\langle n, \varphi\rangle \stackrel{L}{\hookrightarrow} m$ and $\varphi_{B}: m \stackrel{L}{\longleftarrow}\langle n, \varphi\rangle$ denote the forward and backward projections, respectively, of the pair $\langle n, \varphi\rangle$ onto $m$. The following hold for state-set projections:

(1) If an execution starting from a state $s \in \llbracket \varphi \rrbracket$ at node $n$ reaches node $m$ with state $t$, then $t \in \llbracket \varphi_{F} \rrbracket$.

(2) If an execution starting from node $m$ with state $t$ reaches node $n$ with state $s \in \llbracket \varphi \rrbracket$ then $t \in \llbracket \varphi_{B} \rrbracket$.

\subsection{Infeasibility-Type Theorems}

The state-set projections computed using forward and backward propagation can be used to detect semantically infeasible paths in a CFG. Let $n_{1}, \ldots, n_{k}$ be a subset of nodes in the CFG, $n_{0}$ be the initial node and $n_{k+1}$ be some target node of interest. We wish to find if an execution may reach $n_{k+1}$ starting from $n_{0}$, while passing through each of the nodes $n_{1}, \ldots, n_{k}$, possibly more than once and in an arbitrary order. Let $\Pi\left(n_{0}, \ldots, n_{k+1}\right)$ denote the set of all such syntactically valid paths in the CFG.

Let $\varphi_{i}:\left\langle n_{i}\right.$, true $\rangle \stackrel{L}{\hookrightarrow} n_{k+1}, i \in[0, k+1]$, denote the forward state-set projections from $\left\langle n_{i}\right.$, true $\rangle$ onto the final node $n_{k+1}$. Similarly, let $\psi_{i}: n_{0} \stackrel{L}{\longleftarrow}\left\langle n_{i}\right.$, true $\rangle$, $i \in[0, k+1]$, denote the backward projections from $\left\langle n_{i}\right.$, true $\rangle$ onto node $n_{0}$.

Theorem 1 (Infeasibility-type theorem). The paths in $\Pi\left(n_{0}, \ldots, n_{k+1}\right)$ are all semantically infeasible if either

1. $\varphi_{0} \wedge \varphi_{1} \wedge \cdots \wedge \varphi_{k} \wedge \varphi_{k+1} \equiv \emptyset$, or

2. $\psi_{0} \wedge \psi_{1} \wedge \cdots \wedge \psi_{k} \wedge \psi_{k+1} \equiv \emptyset$.

Proof. The proof uses facts about the forward and backward state-set projections. From Lem. 1, we conclude that any path that reaches $n_{k+1}$ starting from $\left\langle n_{i}\right.$, true $\rangle$ must do so with a state that satisfies $\varphi_{i}$. As a result, consider a path that traverses all of $n_{0}, \ldots, n_{k}$ to reach $n_{k+1}$. The state at node $n_{k+1}$ must simultaneously satisfy $\varphi_{0}, \varphi_{1}, \ldots, \varphi_{k}$. Since the conjunction of these assertions is empty, we conclude that no such path may exist.

A similar reasoning applies to backward projection from node $n_{i}$ to node $n_{0}$ using the assertions $\psi_{0}, \ldots, \psi_{k+1}$. 
It is also possible to formulate other infeasibility-type theorems that are similar to Thm. 1 using state-set projection. Let $\eta_{F}$ be the forward fixpoint map computed starting from $\left\langle n_{0}, \varphi_{0}\right\rangle$. Let $\psi_{i}: n_{0} \stackrel{L}{\longleftarrow}\left\langle n_{i}, \eta_{F}\left(n_{i}\right)\right\rangle$ be the state-set projection of the set $\eta_{F}\left(n_{i}\right)$ from node $n_{i}$ onto node $n_{0}$.

Lemma 2. If $\psi_{1} \wedge \ldots \wedge \psi_{k} \wedge \psi_{k+1} \equiv \emptyset$ then there is no semantically valid path from node $n_{0}$ to node $n_{k+1}$ that passes through all of $n_{1}, \ldots, n_{k}$.

A similar result can be stated for a pair of nodes using the forward and the backward fixpoint maps. Let $\left\langle n_{E}, \varphi_{E}\right\rangle$ be an error configuration of interest, $\eta_{F}$ be the forward (post) fixpoint map computed starting from $\left\langle n_{0}, \varphi_{0}\right\rangle$ and $\eta_{B}$ be the backward (post) fixpoint map computed starting from $\left\langle n_{E}, \varphi_{E}\right\rangle$.

Lemma 3. Any error trace that leads to a state in the error configuration $\left\langle n_{E}, \varphi_{E}\right\rangle$ cannot visit node $n^{\prime}$ if $\eta_{F}\left(n^{\prime}\right) \wedge \eta_{B}\left(n^{\prime}\right) \equiv \emptyset$.

Example 2. Consider the example program shown in Fig. 1. We wish to prove the infeasibility of any path that simultaneously visits $n_{0}, n_{2}$ and $n_{4}$. Using state-set projections, we obtain

$$
\begin{aligned}
& \psi_{2}: n_{0} \stackrel{L}{\leftrightarrows}\left\langle n_{2}, \text { true }\right\rangle=\{x \mid x \leq 0\} \\
& \psi_{4}: n_{0} \stackrel{L}{\longleftarrow}\left\langle n_{4}, \text { true }\right\rangle=\{x \mid x>0\}
\end{aligned}
$$

Since $\psi_{2}$ and $\psi_{4}$ are disjoint, it is not possible for an execution of the CFG to visit simultaneously the nodes $n_{0}, n_{2}$ and $n_{4}$. Likewise, a semantically valid path cannot visit $n_{2}$ and $n_{4}$ simultaneously, since $\varphi_{2}:\left\langle n_{2}\right.$, true $\rangle \stackrel{L}{\hookrightarrow} n_{4} \equiv \emptyset$.

\subsection{Infeasible-Path Enumeration}

Thus far, we can detect if all the program paths in $\Pi\left(n_{0}, \ldots, n_{k+1}\right)$ are semantically infeasible for a given set $n_{1}, \ldots, n_{k}, n_{k+1}$. We now consider the problem of enumerating such sets using the results derived in the previous sections. Let $N=\left\{n_{0}, n_{1}, \ldots, n_{m}\right\}$ denote the set of all nodes in the CFG. In order to apply infeasibility-type theorems such as Thm. 1 and Lem. 2, we compute $m+1$ stateset projections $\psi_{0}, \ldots, \psi_{m}$ corresponding to the nodes $n_{0}, \ldots, n_{m}$, respectively. Furthermore, to test the subset $\left\{n_{i_{1}}, \ldots, n_{i_{k}}\right\} \subseteq N$, we test the conjunction $\psi_{i_{1}} \wedge \cdots \wedge \psi_{i_{k}}$ for satisfiability. Therefore, to enumerate all such subsets, we need to enumerate all index sets $I \subseteq\{1, \ldots, m\}$ such that $\bigwedge_{i \in I} \psi_{i} \equiv$ false. For each such set $I$, the corresponding subset of $N$ characterizes the infeasible paths.

Definition 2 (Infeasible \& Saturated Index Set). Given assertions $\varphi_{1}$, $\ldots, \varphi_{m}$, an index set $I \subseteq\{1, \ldots, m\}$ is said to be infeasible iff $\bigwedge_{j \in I} \varphi_{j} \equiv$ false. Likewise, an infeasible index set $I$ is said to be saturated iff no proper subset is itself infeasible.

Note that each infeasible set is an unsatisfiable core of the assertion $\varphi_{1} \wedge$ $\cdots \wedge \varphi_{m}$. Each saturated infeasible set is a minimal unsatisfiable core w.r.t 
set inclusion. Given assertions $\varphi_{1}, \ldots, \varphi_{m}$, we wish to enumerate all saturated infeasible index sets. To solve this problem, we provide a generic method using a SAT solver to aid in the enumeration. This method may be improved by encoding the graph structure as a part of the SAT problem to enumerate continuous segments in the CFG. We also present domain-specific techniques for directly enumerating all the cores without using SAT solvers.

Generic Enumeration Technique. Assume an oracle $O$ to determine if a conjunctive theory formula $\psi_{I}: \bigwedge_{i \in I} \psi_{i}$ is satisfiable. Given $O$, if $\psi_{I}$ is unsatisfiable we may extract a minimal unsatisfiable core set $J \subseteq I$ as follows: (1) set $J=I$, (2) for some $j \in J$, if $\psi_{J-\{j\}}$ is unsatisfiable, $J:=J-\{j\}$, and (3) repeat step 2 until no more conjuncts need to be considered. Alternatively, $O$ may itself be able to provide a minimal core.

Our procedure maintains a family of subsets $\mathfrak{I} \subseteq 2^{\{1, \ldots, m\}}$ that have not (yet) been checked for feasibility. Starting from $\mathfrak{I}=2^{\{1, \ldots, m\}}$, we carry out steps (a) and (b), below, until $\mathfrak{I}=\emptyset$ :

(a) Pick an untested subset $J \in \mathfrak{I}$.

(b) Check the satisfiability of $\psi_{J}: \bigwedge_{j \in J} \varphi_{j}$.

If $\psi_{J}$ is satisfiable, then remove all subsets of $J$ from $\mathfrak{I}: \mathfrak{I}^{\prime}=\mathfrak{I}-\{K \mid K \subseteq$ $J\}$. If $\psi_{J}$ is unsatisfiable, compute a minimal core set $C \subseteq J$. Remove all supersets of $C: \mathfrak{I}^{\prime}=\mathfrak{I}-\{I \mid I \supseteq C\}$. Also, output $C$ as an infeasible set.

Symbolic enumeration using SAT. In practice, the set $\mathfrak{I}$ may be too large to maintain explicitly. It is therefore convenient to encode it succinctly in a SAT formula. We introduce Boolean selector variables $y_{1}, \ldots, y_{m}$, where $y_{i}$ denotes the presence of the assertion $\varphi_{i}$ in the theory formula. The set $\mathfrak{I}$ is represented succinctly by a Boolean formula $\mathfrak{F}$ over the selector variables. The initial formula $\mathfrak{F}_{0}$ is set to true. At each step, we may eliminate all supersets of a set $J$ by adding the new clause $\bigvee_{j \in J} \neg y_{j}$. Fig. 2 shows the procedure to enumerate all infeasible indices using SAT solvers and elimination of unsatisfiable cores.

Graph-based enumeration using SAT. To further improve the enumeration procedure, we note that many subsets $I \subseteq N$ may not lead to any syntactically valid paths through the CFG that visit all nodes in $I$. Such subsets need not be considered. Nodes $n_{i}$ and $n_{j}$ are said to conflict if there is no syntactic path starting from $n_{0}$ that visits both $n_{i}$ and $n_{j}$. Let $C \subseteq N \times N$ denote the set of all conflicting node pairs. We exclude conflicting nodes or their supersets from the enumeration process by adding the clause $\neg y_{i} \vee \neg y_{j}$ for each conflict pair $\left(n_{i}, n_{j}\right) \in C$. However, in spite of adding the conflict information, syntactically meaningless subsets may still be enumerated by our technique.

Example 3. Consider the CFG skeleton in Fig. 1, disregarding the actual operations in its nodes and edges. We suppose that all paths between nodes $n_{0}$ and $n_{5}$ are found to be infeasible: i.e., $\{0,5\}$ is an infeasible index set. Clearly, due to the structure of the CFG, there is now no need to check the satisfiability of the index set $\{0,3\}$, since all paths to node $n_{5}$ have to pass through node $n_{3}$. 


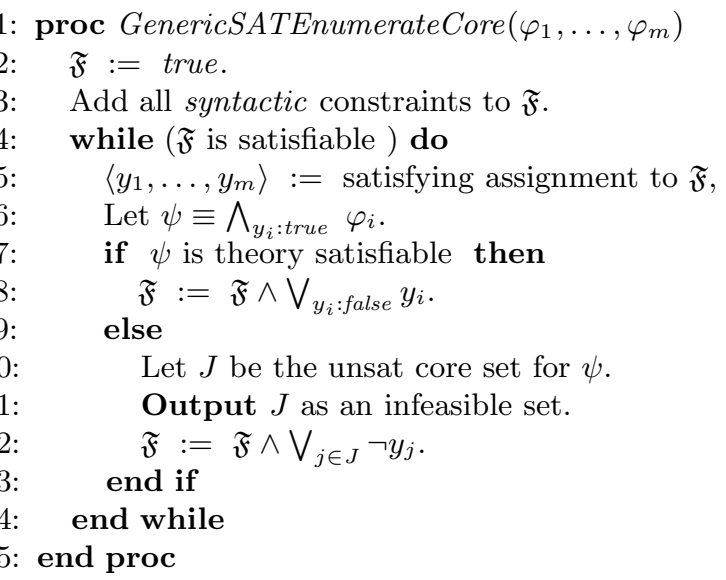

Fig. 2. SAT-based enumeration modulo theory to enumerate infeasible index sets.

However, this information is not available to the SAT solver, which generates the candidate index set $\{0,3\}$.

To avoid such paths, we restrict the SAT solver to enumerate continuous segments in the CFG.

Definition 3 (Continuous Segment). A subset $I \subseteq N$ is a continuous segment iff for each $n_{i} \in I$, some successor of $n_{i}$ (if $n_{i}$ has any successors) and some predecessor (if $n_{i}$ has any predecessors) belong to $I$.

An infeasible index set $I$ is syntactically meaningful iff there exists a continuous segment $C$ such that $I \subseteq C$. Therefore, it suffices to restrict our SAT solver to enumerate all feasible continuous segments $C$ in the CFG. The unsatisfiable core set for any such segment is also a syntactically meaningful set. Secondly, if a continuous segment $C$ is shown to be semantically infeasible by a subset $I$, we are not interested in other infeasible subsets $I^{\prime}$ that show $C$ to be infeasible.

Let $p_{1}, \ldots, p_{m}$ be the indices of predecessors of a node $n_{i}(m \geq 1)$, and $s_{1}, \ldots, s_{r}$ denote the successors indices $(r \geq 1)$. We encode continuous segments by adding the following constraints, corresponding to each node $n_{i}$ in the CFG:

- Forward: If $m>0$, add $\neg y_{i} \vee y_{p_{1}} \vee y_{p_{2}} \vee \ldots \vee y_{p_{m}}$.

- Backward: If $r>0$, add $\neg y_{i} \vee y_{s_{1}} \vee y_{s_{2}} \vee \ldots \vee y_{s_{r}}$.

The total size of these constraints is linear in the size of the CFG (number of nodes, number of edges). Fig. 2 is modified to add the continuous segment constraints to $\mathfrak{F}$, in addition to the conflicts.

Example 4. Again considering the CFG skeleton from Fig. 1, if the index set $\{0,5\}$ is found for the unsatisfiable core of the continuous segment $C:\{0, \ldots, 5\}$, the additional clause $\neg y_{0} \vee \neg y_{5}$ will prevent any future consideration of this segment. The index set $\{0,3\}$ will not be considered, because, $n_{3}$ is also part of the continuous segment $C$ which has already been shown to be infeasible. 
Utilizing SMT and MAX-SAT Techniques. In principle, a tighter integration of the propositional and theory part can be obtained by enumerating all minimal unsatisfiable cores of an SMT (Satisfiability Modulo Theories) formula $\Psi_{m}: \wedge_{i=0}^{m}\left(\left(\neg y_{i} \vee \varphi_{i}\right) \wedge\left(y_{i} \vee \neg \varphi_{i}\right)\right)$, along with other propositional clauses over $y_{1}, y_{2}, \ldots, y_{m}$ arising from conflict pairs and syntactic graph-based constraints discussed earlier.

The problem of finding all minimal unsatisfiable cores is related to the problem of finding all maximal satisfiable solutions ${ }^{4}[2,15]$. This duality has been exploited to use procedures for finding maximal satisfiable solutions (MAX-SAT) for generating all minimal unsatisfiable cores. This could lead to improved performance, since checking satisfiability is usually considered easier in practice than checking unsatisfiability.

Enumerating Unsatisfiable Cores Directly. In some domains, it is possible to directly enumerate all the unsatisfiable cores of the conjunction $\varphi_{1} \wedge \varphi_{2} \wedge \cdots \wedge$ $\varphi_{m}$. Each unsatisfiable core directly yields infeasible index sets. The advantage of this enumeration method is that it avoids considering index sets for which the corresponding conjunctions is theory satisfiable. Secondly, the properties of the underlying abstract domain can be exploited for efficient enumeration. The disadvantage is that the same infeasible index sets may be repeatedly obtained for different unsatisfiable cores.

As a case in point, we consider the interval domain. The concretizations of interval domain objects are conjunctions of the form $x_{i} \in\left[l_{i}, u_{i}\right]$. Let $\varphi_{1}, \ldots, \varphi_{m}$ be the result of the state projections carried out using interval analysis. We assume that each $\varphi_{i}$ is satisfiable. Let $\varphi_{i}$ be the assertion $\bigwedge_{j} x_{j} \in\left[l_{i j}, u_{i j}\right]$, wherein each $l_{i j} \leq u_{i j}$. The lack of relational information in the interval domain restricts each unsatisfiable core to be of size at most 2 :

Lemma 4. Any unsatisfiable core in $\bigwedge_{i} \varphi_{i}$ involves exactly two conjuncts: $l_{i j} \leq$ $x_{j} \leq u_{i j}$ in $\varphi_{i}$ and $l_{k j} \leq x_{j} \leq u_{k j}$ such that $\left[l_{i j}, u_{i j}\right] \cap\left[l_{k j}, u_{k j}\right]=\emptyset$.

As a result, it is more efficient to enumerate infeasible paths using domainspecific reasoning for the interval domain. Direct enumeration of unsatisfiable cores is possible in other domains also. For instance, we may enumerate all the negative cycles for the octagon domain, or all dual polyhedral vertices in the polyhedral domain.

\section{Path-Sensitive Analysis}

In this section, we describe how information about infeasible paths discovered in $\S 3$ can be used to improve the accuracy of a path-insensitive analysis.

Example 5. Consider the program shown in Fig. 3. Clearly, the assertion at line 9 in the program is never violated. However, neither a forward propagation nor

\footnotetext{
${ }^{4}$ Specifically, the set of all minimal unsatisfiable cores, also called MUS-es, is equivalent to the set of all irreducible hitting sets of all MCS-es, where each MCS is the complement of a maximal satisfiable solution.
} 

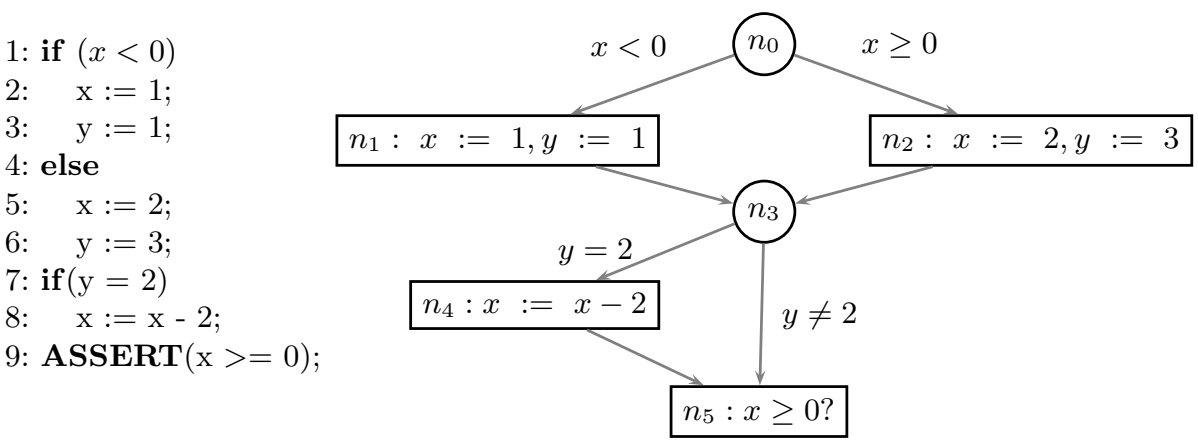

Fig. 3. An example program (left) along with its CFG representation (right). (Node numbers do not correspond to line numbers.)

a backward propagation using the interval domain is able to prove the assertion in node $n_{5}$. A SAT-based infeasible path enumeration using the interval domain enumerates the sets $\left\{n_{2}, n_{4}\right\}$ and $\left\{n_{1}, n_{4}\right\}$ as infeasible.

Syntactic Language Refinement (SLR). Let $\Pi:\left\langle N, E, \mu, n_{0}, \varphi_{0}\right\rangle$ be a CFG with a property $\langle n$, false $\rangle$ to be established. The syntactic language of $\Pi$ consists of all the edge sequences $e_{1}, \ldots, e_{m}$ that may be visited along some walk through the CFG starting from $n_{0}$. In effect, we treat $\Pi$ as an automaton over the alphabet $E$, wherein each edge $e_{i}$ accepts the alphabet symbol $e_{i}$. Let $L_{\Pi}$ denote the language of all edge sequences accepted by $\mathrm{CFG} \Pi$, represented as a deterministic finite automaton.

The results of the previous section allow us to infer sets $I:\left\{n_{1}, \ldots, n_{k}\right\} \subseteq N$ such that no semantically valid path from node $n_{0}$ to node $n$ may pass through all the nodes of the set $I$. For each set $I$, we remove all the (semantically invalid) paths in the set $\Pi\left(I \cup\left\{n_{0}, n\right\}\right)$ from the syntactic language of the CFG:

$$
L_{\Pi}^{\prime}=L_{\Pi}-\underbrace{\left\{\pi \mid \pi \text { is a path from } n_{0} \text { to } n, \text { passing through all nodes in } I\right\}}_{L_{I}}
$$

Since the sets $L_{\Pi}$ and $L_{I}$ are regular, $L_{\Pi^{\prime}}=L_{\Pi}-L_{I}$ is also regular. The refinement procedure continues to hold even if $L_{\Pi}$ is context free, which happens in the presence of function calls and returns in the CFG.

Let $\Pi^{\prime}$ be the automaton recognizing $L_{\Pi^{\prime}}$. The automaton $\Pi^{\prime}$ can be viewed as a CFG once more by associating actions (conditions and assignments) with its edges. Each edge labeled with the alphabet $e_{i}$ is provided the action $\mu\left(e_{i}\right)$ from the original CFG $\Pi$. Since $L_{\Pi^{\prime}} \subseteq L_{\Pi}, \Pi^{\prime}$ encodes a smaller set of syntactically valid paths. Therefore, abstract interpretation on $\Pi^{\prime}$ may result in a more precise fixpoint.

Example 6. Consider the CFG skeleton $\Pi$ in Fig. 4(a). Suppose nodes $\left\{n_{4}, n_{8}\right\}$ were found to be infeasible. The refined CFG $\Pi^{\prime}$ is shown in Fig. 4(b). The 


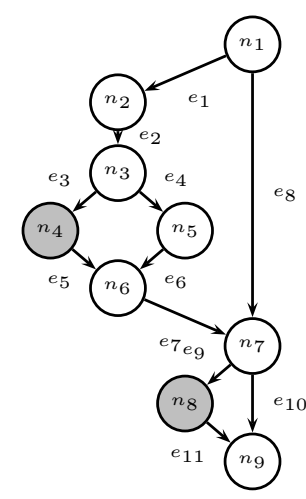

(a)

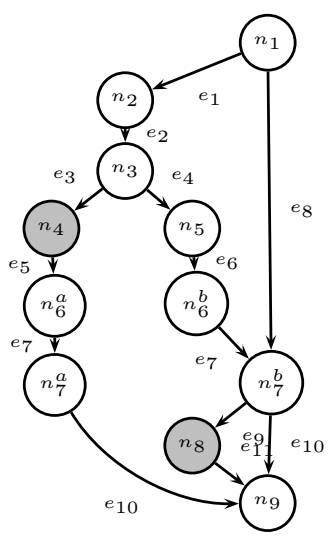

(b)

Fig. 4. Path-sensitivity can be simulated by syntactic language refinement: (a) original CFG, (b) paths visiting nodes $\left\{n_{4}, n_{8}\right\}$ are found infeasible and removed.

edges and the nodes are labeled to show the correspondences between the two CFGs. Notice that it is no longer possible to visit the shaded nodes in the same syntactic path.

A path sensitive static analysis might be able to obtain the same effect by not using join at node $n_{6}$, and partially merging two disjuncts at node $n_{7}$. Borrowing the terminology from our previous work the CFG $\Pi^{\prime}$ is an elaboration of the CFG $\Pi[22]$, or from the terminology of Mauborgne et al., a trace partitioning [13, 17], that keeps the trace visiting node $n_{4}$ separate from the other traces. However, our prior knowledge of the infeasibility of $\left\{n_{4}, n_{8}\right\}$ enables us to automatically rule out the edge $n_{7}^{a} \rightarrow n_{8}$.

From the discussion above, it is evident that syntactic language refinement can be cast in the framework of related schemes such as elaborations or tracepartitions. However, the key difference is that our scheme always uses the infeasible CFG paths as a partitioning heuristic. The heuristic used in the elaboration or trace-partitioning may be unable to guess the right partitions required to detect the infeasible paths in practice.

Example \%. Returning to the example in Fig. 3, we find that the paths from $n_{0} \rightsquigarrow n_{5}$, traversing the nodes $I_{0}:\left\{n_{1}, n_{4}\right\}$ and $I_{1}:\left\{n_{2}, n_{4}\right\}$ are semantically infeasible. Therefore, we may remove such paths from the CFG using syntactic language refinement. The resulting CFG $\Pi^{\prime}$ is simply the original CFG with the node $n_{4}$ removed. A path-insensitive analysis over $\Pi^{\prime}$ proves the property.

In theory, it is possible to first remove infeasible path segments using abstract interpretation, perform a language refinement, and subsequently, analyze the refined CFG. In practice, however, we observe that most infeasible paths involve no more than two intermediate nodes. Furthermore, the size of the refined CFG after a set $I$ has been removed can be a factor $2^{|I|}$ larger. 


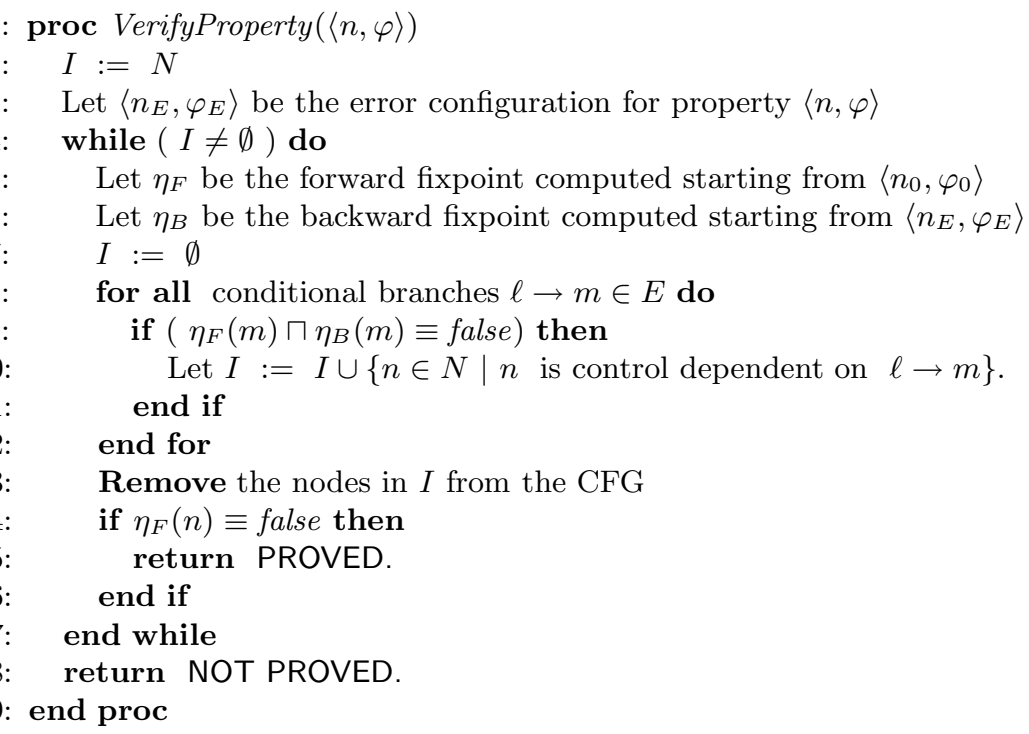

Fig. 5. Using infeasible-path detection to improve path-insensitive analysis.

Application. We now present a simple version of the SLR scheme using Lem. 3 that removes at most one intermediate node w.r.t a given property. Secondly, we repeatedly refine the CFG at each stage by using the improved abstract interpretation result on the original CFG. Finally, thanks to the form of Lem. 3, each application of the scheme requires just two fixpoint computations, one in the forward direction and the other in the backward.

Fig. 5 shows an iterative syntactic language refinement scheme. Each step involves a forward fixpoint from the initial node and a backward fixpoint computed from the property node. First, infeasible pairs of nodes are then determined using Lem. 3, and the paths involving such pairs are pruned from the CFG. Since paths are removed from the CFG, subsequent iterations can produce stronger fixpoints, and therefore, detect more infeasible intermediate nodes. The language refinement is repeated until the property is verified, or no new nodes are detected as infeasible in consecutive iterations.

Example 8. VerifyProperty proves the assertion in the example shown in Fig. 3. During the first iteration, the condition at line 8 of VerifyProperty holds for the edge $n_{0} \rightarrow n_{2}$. Consequently, node $n_{2}$ will be removed before the next forward fixpoint computation. Hence, interval analysis will be able to determine that edge $n_{3} \rightarrow n_{4}$ is infeasible, and therefore, the property $\left\langle n_{5}, x \geq 0\right\rangle$ is verified. $\otimes$

\section{Experiments}

We have implemented the SLR technique inside the F-SofT C program verifier [14] to check array, pointer, and C string usage. The analyzer is context 
Table 1. Number of saturated infeasible sets from SAT-based enumeration.

\begin{tabular}{|l|r|r|r||r|r|r|}
\hline & & & & \multicolumn{2}{|c|}{ Time (s) } & \\
\cline { 4 - 6 } Prog. & LOC & \#Vars & \#Branches & FixPoint & Enum & \#Inf.Sets \\
\hline ex1 & 35 & 7 & 13 & 0.07 & 0.01 & 3 \\
ex2 & 40 & 6 & 15 & 0.08 & 0.02 & 10 \\
ex3 & 79 & 9 & 36 & 1.46 & 0.12 & 6 \\
ex4 & 85 & 71 & 41 & 2160.67 & 6.12 & 0 \\
ex5 & 94 & 12 & 40 & 2.85 & 3.60 & 38 \\
ex6 & 102 & 38 & 27 & 51.76 & 0.15 & 2 \\
ex7 & 115 & 2 & 31 & 0.06 & 0.02 & 28 \\
\hline
\end{tabular}

sensitive, by using call strings to track contexts. Our abstract interpreter supports a combination of different numerical domains, including constant folding, interval, octagon [18], polyhedron [12] and the symbolic range domains [21]. The experiments were run on a Linux machine with two Xeon $2.8 \mathrm{GHz}$ processors and $4 \mathrm{~GB}$ of memory.

Infeasible-Path Enumeration. We implemented the algorithm in Fig. 2 using the octagon abstract domain as a proof-of-concept. Tab. 1 shows the performance of the infeasible-path enumerator over a set of small but complex functions written in $\mathrm{C}$ [23]. As an optimization, we modified the algorithm to enumerate infeasible sets solely involving conditional branches. The running time of the algorithm is a function of the number of conditional branches and the number of variables in the program. Surprisingly, computing fixpoints accounts for the majority of the running time. Not surprisingly, almost all saturated infeasible sets involved exactly two edges. With the additional optimizations described here and a variable-packing heuristic, we hope to scale this technique to larger functions.

Syntactic Language Refinement. We implemented the VerifyProperty algorithm on top of our existing abstract interpreter. Given a program, we first run a series of path-insensitive analyses. Properties thus proved are sliced away from the CFG. The resulting simplified CFG is fed into our analysis. Our analysis is run twice: first, using the interval domain, and then using the octagon domain on the sliced CFG from the interval analysis instantiation. Tab. 2 shows the performance of our tool chain on a collection of industrial as well as open source projects. For each program, we show the number of proofs obtained and the time taken by the base analysis as well as the additional proofs along with the overhead of the SLR technique using the intervals and the octagon domains successively. For our set of examples, the SLR technique obtains $15 \%$ more proofs over and above the base analysis. However, it involves a significant time overhead of roughly $15 \%$ for the interval domain and $75 \%$ for the octagon domain. A preliminary comparison with our previous work suggests that this overhead is quite competitive with related techniques for performing path-sensitive analysis [22].

Tab. 3 shows a direct comparison of our implementation with a partially path-sensitive analysis implemented using CFG elaborations [22]. The compari- 
Table 2. Performance of the tool flow using SLR. $\left(H_{i}\right.$ : mobile software application modules, $L_{i}$ : Linux device drivers, $M_{i}$ : a network protocol implementation modules.)

\begin{tabular}{|c|c|c|c|c|c|c|c|}
\hline \multirow[b]{3}{*}{ Code } & \multirow{3}{*}{$\begin{array}{c}\text { Simplified } \\
\text { LOC }\end{array}$} & \multicolumn{2}{|c|}{ Base analysis } & \multicolumn{4}{|c|}{ Additional Proofs with SLR } \\
\hline & & & & $\overline{\text { Inter }}$ & vals & Octa & gons \\
\hline & & Proofs & Time $(\mathrm{s})$ & Proofs & Time $(\mathrm{s})$ & Proofs & Time $(\mathrm{s})$ \\
\hline$H_{1}$ & 2282 & $7 / 14$ & 28.08 & $0 / 7$ & 0.5 & $0 / 7$ & 8.77 \\
\hline$H_{2}$ & 3319 & $33 / 49$ & 355.85 & $0 / 16$ & 7.04 & $0 / 16$ & 102.24 \\
\hline$H_{3}$ & 2668 & $23 / 37$ & 52.49 & $0 / 14$ & 1.25 & $0 / 14$ & 14.17 \\
\hline$L_{4}$ & 4626 & $12 / 31$ & 10.21 & $0 / 19$ & 5.07 & $0 / 19$ & 9.1 \\
\hline$M_{5}$ & 5095 & $67 / 265$ & 69.62 & $35 / 198$ & 84.99 & $28 / 163$ & 217.44 \\
\hline$L_{6}$ & 5346 & $6 / 20$ & 3.62 & $0 / 14$ & 1.53 & $0 / 14$ & 7.59 \\
\hline$L_{7}$ & 5599 & $5 / 146$ & 681.48 & $0 / 141$ & 198.26 & $0 / 141$ & 239.39 \\
\hline$M_{8}$ & 6142 & $109 / 314$ & 86.85 & $1 / 205$ & 173.74 & $98 / 204$ & 2008.07 \\
\hline$L_{9}$ & 6326 & $119 / 135$ & 70.88 & $0 / 16$ & 2.74 & $0 / 16$ & 8.18 \\
\hline$M_{10}$ & 6645 & $93 / 385$ & 244.57 & $25 / 292$ & 390.15 & $70 / 267$ & 990.11 \\
\hline$M_{11}$ & 7541 & $162 / 442$ & 262.94 & $77 / 280$ & 361.08 & $49 / 203$ & 1069.1 \\
\hline$M_{12}$ & 10206 & $285 / 745$ & 1479.29 & $30 / 460$ & 1584.45 & $154 / 430$ & 6681.36 \\
\hline$M_{13}$ & 11803 & $325 / 786$ & 1089.86 & $121 / 461$ & 859.36 & $99 / 340$ & 5710.56 \\
\hline$L_{14}$ & 13162 & $38 / 114$ & 289.9 & $34 / 76$ & 130.98 & $0 / 42$ & 263.86 \\
\hline$M_{15}$ & 14665 & $313 / 606$ & 117.96 & $163 / 293$ & 112.19 & $10 / 130$ & 204.16 \\
\hline$M_{17}$ & 26758 & 1904/1918 & 2208.85 & $0 / 14$ & 0.95 & $0 / 14$ & 8.89 \\
\hline$M_{18}$ & 47213 & $4173 / 4218$ & 16880.00 & $21 / 45$ & 4.07 & $0 / 24$ & 20.51 \\
\hline Total & & $7674 / 10225$ & & $507 / 2551$ & & $508 / 2044$ & \\
\hline
\end{tabular}

Table 3. Comparison of SLR with path-sensitive analysis using CFG elaborations [22]. (\#T: total time with two outlying data points removed, \#P: additional proofs.)

\begin{tabular}{|l|r|r|r|r|r|r|r|r|r|}
\hline \multirow{4}{*}{ \#Progs } & \multicolumn{3}{|c|}{ Base } & \multicolumn{3}{c|}{ CFG } & \multicolumn{4}{c|}{ SLR } \\
\cline { 2 - 9 } & Tot. & Proofs & \#T & \#P & \#T & \#P & \#T & \#P & \#T \\
\hline 48 & 403 & 178 & 2.47 & +45 & 76.42 & +44 & 27 & +79 & 36 \\
\hline
\end{tabular}

son is carried out over a collection of small example programs [23] written in the C language. These programs range from 20-400 lines of code, and are designed to evaluate the handling of loops, aliasing, dynamic allocation, type-casts, string library functions and other sources of complexities in practical programs. Because the latter implementation is context-insensitive, we compare against a contextinsensitive version of our technique as well. CFG elaboration technique achieves 45 extra proofs with 50x time overhead. Our context-insensitive implementation proves roughly as many properties as CFG elaborations with a much smaller overhead (roughly 10x for context-insensitive and 20x for context-sensitive). Our implementation proved quite a few properties that could not be established by elaborations and vice versa. Not surprisingly, added context-sensitivity to our technique renders it vastly superior. 


\section{References}

1. R. Bagnara, P. M. Hill, and E. Zaffanella. Widening operators for powerset domains. STTT, 9(3-4):413-414, 2007.

2. J. Bailey and P. J. Stuckey. Discovery of minimal unsatisfiable subsets of constraints using hitting set dualization. In PADL, 2005.

3. R. Bodik, R. Gupta, and M. L. Soffa. Refining data flow information using infeasible paths. In FSE, pages 361-377. Springer-Verlag, 1997.

4. François Bourdoncle. Abstract debugging of higher-order imperative languages. In PLDI '93, pages 46-55. ACM, 1993.

5. P. Cousot and R. Cousot. Abstract interpretation: A unified lattice model for static analysis of programs by construction of approximation of fixed points. In Proc. Principles of Programming Languages (POPL), 1977.

6. P. Cousot and R. Cousot. Static determination of dynamic properties of recursive procedures. In Formal Descriptions of Programming Concepts. North-Holland, 1978.

7. P. Cousot, P. Ganty, and J-F. Raskin. Fixpoint-guided abstraction refinements. In $S A S$, volume 4634 of $L N C S$, pages 333-348, 2007.

8. M. Das, S. Lerner, and M. Seigle. ESP: path-sensitive program verification in polynomial time. In PLDI, pages 57-68. ACM Press, 2002.

9. D. Dhurjati, M. Das, and Y. Yang. Path-sensitive dataflow analysis with iterative refinement. In $S A S$, pages 425-442, 2006.

10. J. Fischer, R. Jhala, and R. Majumdar. Joining dataflow with predicates. In FSE, 2005.

11. B. S. Gulavani and S. K. Rajamani. Counterexample driven refinement for abstract interpretation. In TACAS, volume 3920 of $L N C S$, pages 474-488, 2006.

12. N. Halbwachs, Y.-E. Proy, and P. Roumanoff. Verification of real-time systems using linear relation analysis. Formal Methods in Sys. Design, 11(2):157-185, 1997.

13. Maria Handjieva and Stanislav Tzolovski. Refining static analyses by trace-based partitioning using control flow. In $S A S$, volume 1503 of $L N C S, 1998$.

14. F. Ivančić, I. Shlyakhter, A. Gupta, M. K. Ganai, V. Kahlon, C. Wang, and Z. Yang. Model checking C programs using F-SOFT. In ICCD, pages 297-308, 2005.

15. Mark H. Liffiton and Karem A. Sakallah. Algorithms for computing minimal unsatisfiable subsets of constraints. J. of Automated Reasoning, 40(1):133, 2008.

16. R. Manevich, S. Sagiv, G. Ramalingam, and J. Field. Partially disjunctive heap abstraction. In $S A S$, volume 3148 of $L N C S$, pages 265-279, 2004.

17. L. Mauborgne and X. Rival. Trace partitioning in abstract interpretation based static analyzers. In European Symp. on Programming (ESOP), 2005.

18. A. Miné. The octagon abstract domain. In Working Conf. on Reverse Eng., 2001.

19. Minh Ngoc Ngo and Hee Beng Kuan Tan. Detecting large number of infeasible paths through recognizing their patterns. In FSE, 2007.

20. Xavier Rival. Understanding the origin of alarms in ASTRÉE. In SAS, 2005.

21. S. Sankaranarayanan, F. Ivančić, and A. Gupta. Program analysis using symbolic ranges. In $S A S$, pages 366-383, 2007.

22. S. Sankaranarayanan, F. Ivančić, I. Shlyakhter, and A. Gupta. Static analysis in disjunctive numerical domains. In $S A S$, volume 4134 of $L N C S$, pages 3-17, 2006.

23. Sriram Sankaranarayanan. NECLA static analysis benchmarks (2007). Available from http://www.nec-labs. com/ f soft. 